\title{
XMM-Newton study of the very young stellar cluster IC 348
}

\author{
T. Preibisch ${ }^{1}$ and H. Zinnecker ${ }^{2}$ \\ 1 Max-Planck-Institut für Radioastronomie, Auf dem Hügel 69, 53121 Bonn, Germany \\ e-mail: preib@mpifr-bonn.mpg.de \\ 2 Astrophysikalisches Institut Potsdam, An der Sternwarte 16, 14482 Potsdam, Germany \\ e-mail: hzinnecker@aip.de
}

Received 6 February 2004 / Accepted 8 April 2004

\begin{abstract}
We analyze a XMM-Newton X-ray imaging observation of the very young stellar cluster IC 348 with an observing time of $12 \mathrm{~h}$ and a corresponding total (all detector) MOS-equivalent exposure time of $207 \mathrm{ks}$. Our observation is strongly affected by a very intense solar particle flare, due to which only the first half of the exposure time can be used for scientific analysis. We compare the X-ray sources seen by XMM-Newton with those found in our previous Chandra study of IC 348 and find that XMM-Newton reveals 71 new X-ray sources, most of which are located outside the field-of-view of the Chandra observation. 20 of these new X-ray sources can be identified with known cluster members, and 19 sources are likely to be new low-mass members of the cluster. The lightcurves of the XMM-Newton sources show at most moderate levels of variability, but no large flares. We compare the spectral fitting results for 10 stars for which we have good spectra from both Chandra and $X M M$-Newton, and find good agreement in the fitted plasma temperatures, but a systematic difference in the fitted values of the hydrogen column density (extinction). We finally discuss the X-ray properties of the optically bright B1 star $o$ Per and the X-ray detections among the deeply embedded young stellar objects to the south of the optical cluster center (including HH 211-mm), and find that none of the 23 spectroscopically identified brown dwarfs in IC 348 is detected in our XMM-Newton data.
\end{abstract}

Key words. Galaxy: open clusters and associations: individual: IC 348 - stars: formation - stars: coronae stars: low-mass, brown dwarfs - stars: pre-main sequence - X-rays: stars

\section{Introduction}

IC 348 is a very young stellar cluster in the Perseus molecular cloud complex, associated with the Per OB2 association and located at a distance of about 310 pc (cf. Herbig 1998). The cluster is exceptionally well investigated in optical and infrared wavelengths. Several studies have identified cluster members by extensive spectroscopy and photometry at optical and IR wavelengths (cf. Herbig 1998; Lada \& Lada 1995; Luhman et al. 1998; Luhman 1999) or narrow-band photometry (Najita et al. 2000). Studies of the infrared luminosity function have provided information on the stellar and sub-stellar mass function (Lada et al. 1998; Muench et al. 2003; Preibisch et al. 2003). Recently, Luhman et al. (2003) presented a new census of the stellar and substellar members of IC 348. From spectroscopy of candidate cluster members they identified numerous new members, including several brown dwarfs. Their final, extinction-limited $\left(A_{\mathrm{V}} \leq 4 \mathrm{mag}\right)$ sample of members is unbiased in mass, nearly complete down to masses of $M \geq 0.03 M_{\odot}$, and contains 288 spectroscopically identified cluster members, including 23 brown dwarfs.

The basic properties of IC 348 can be summarized as follows: the mean age of the cluster members is $\sim 2 \mathrm{Myr}$ and most of the stars exhibit relatively low extinction $\left(A_{\mathrm{V}} \lesssim 4\right.$ mag). The majority of the cluster members is in the T Tauri stage of premain sequence evolution, and more than 50 stars can be classified as "classical T Tauri stars" (CTTS; $W(\mathrm{H} \alpha) \geq 10 \AA)$. In the central parts of the cluster the active star formation phase seems to be finished, and the cluster population represents the outcome of a recent star formation event. About $10^{\prime}$ to the southwest of the cluster center, however, a dense cloud core is found which contains several deeply embedded infrared sources with extinctions exceeding $\sim 20 \mathrm{mag}$ in $A_{\mathrm{V}}$ as well as the very young molecular hydrogen jet HH 211 (McCaughrean et al. 1994) and the IC 348 MMS outflow (Eislöffel et al. 2003). The star formation process is still underway in this region.

The first X-ray observations of IC 348 were performed with the ROSAT observatory and presented by Preibisch et al. (1996). The ROSAT studies led to the discovery of 116 X-ray sources in a $2^{\circ}$ diameter field of view. We have also obtained a deep X-ray image of IC 348 with the Advanced CCD Imaging Spectrometer (ACIS) on board the Chandra $X$-Ray Observatory, in which we detected 215 individual X-ray sources. The basic results of this observation were reported in Preibisch \& Zinnecker (2001, PZ01 hereafter), and in Preibisch \& Zinnecker (2002, PZ02 hereafter) we studied the X-ray 
properties of the individual young stellar and substellar objects in IC 348. This paper describes a new X-ray study of IC 348 with the XMM-Newton observatory. The aim was to utilize the high sensitivity of XMM-Newton, especially for harder X-rays (5-10 keV), to reveal even fainter X-ray faint sources, in particular among the most strongly obscured cluster members.

\section{Observations and data analysis}

The observations discussed in this paper were obtained with the European Photon Imaging Cameras (EPIC) onboard of XMM-Newton. The EPIC system (see Jansen et al. 2001) consists of two identical EPIC-MOS cameras (Turner et al. 2001) and the EPIC-PN camera (Strüder et al. 2001). The cameras were operated with the medium optical blocking filter and in the Full-Frame mode. They provide a $\approx 30^{\prime}$ diameter field-of-view, energy coverage from $\approx 0.15-15 \mathrm{keV}$, and moderate energy resolution $(E / \Delta E \approx 20-50)$. In comparison to Chandra, XMM-Newton offers a considerably larger effective area $\left(2350 \mathrm{~cm}^{2}\right.$ at $1 \mathrm{keV}$ for EPIC compared to $700 \mathrm{~cm}^{2}$ for Chandra/ACIS), especially at high energies $\left(1400 \mathrm{~cm}^{2}\right.$ at $6 \mathrm{keV}$ for EPIC compared to $200 \mathrm{~cm}^{2}$ for Chandra/ACIS), but has a much lower spatial resolution $\left(15^{\prime \prime}\right.$ half-energy width of the XMM-Newton mirror PSF compared to $0.5^{\prime \prime}$ for Chandra).

The XMM-Newton observation of IC 348 was obtained during the satellite revolution number 577 on 2 nd and 3rd February 2003. The exposure times were $39.4 \mathrm{ks}$ for the MOS 1 and the MOS2 camera, and $44.4 \mathrm{ks}$ for the PN camera. The corresponding total MOS-equivalent exposure time is about 207 ks. Unfortunately, our observation was very strongly affected by high, flaring radiation background during the second half of the exposure time (see Fig. 1). This led to extremely high background count rates of up to several 100 counts per second and even caused the PN camera to encounter a "Full Scientific Buffer" error during this observation. The second half of the observation could therefore not be used for scientific analysis. This reduction of the usable exposure time decreased the expected sensitivity for point-source detection by nearly a factor of 2 (see XMM-Newton User Handbook).

Extraction of science products from the Observation Data Files followed standard procedures using the XMM-Newton Science Analysis System (SAS). We performed our data analysis with the most recent SAS version 5.4.1. In our analysis of the individual sources we concentrated on the data of the PN camera, as this detector yields an about 3 times higher signal (countrates) than the MOS cameras.

\subsection{Source detection and identification}

Source detection was performed with the standard SAS detection pipeline on the summed (MOS1 + MOS2 + PN) dataset. A careful inspection of the X-ray images showed that the automatic detection retrieved nearly all visually apparent X-ray sources correctly, but it also "detected" many spurious sources, which were then manually removed from the source list.

The final source list contains 199 X-ray sources. 152 of these are in the field-of-view (FOV) covered by our previous Chandra observation, while 47 sources are located outside the

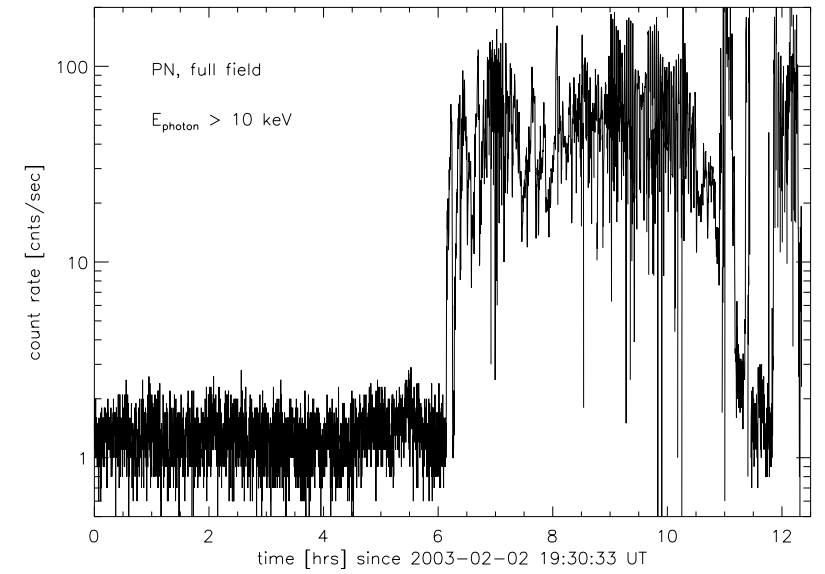

Fig. 1. Background flaring during the XMM-Newton observation, probably due to solar energetic particles. The graph shows the full field EPIC-PN count rate for photons energy in excess of $10 \mathrm{keV}$, which are almost exclusively due to particle background.

Chandra FOV. The next step of our analysis was to compare the XMM-Newton sources in the Chandra FOV to the sources detected by Chandra. We found that the vast majority of the $X M M-N e w t o n$ sources in the common FOV were already detected by Chandra. Furthermore, the rather high stellar density (the typical angular separation of the stars is about $5^{\prime \prime}$ ) in the central part of the cluster led to serious crowding problems due to the rather poor spatial resolution of XMM-Newton $\left(\sim 15^{\prime \prime}\right)$ : most of the apparently single X-ray sources in the $X M M-N e w t o n$ image are in fact resolved into multiple sources in the Chandra image (which provides a much better spatial resolution of $\sim 1^{\prime \prime}$ ). We finally identified 24 XMM-Newton sources which are not already present in the Chandra image. Adding these 24 sources to the 47 sources outside the Chandra FOV, we find that our XMM-Newton observations lead to the detection of 71 new X-ray sources. Table 1 provides a list of these XMM-Newton X-ray sources; we denote them by names in the form XMMU JHHMMSSS.s+DDMMSS based on their J2000 coordinates. For the discussion of those X-ray sources that were already detected by Chandra we use their names of the form CXOPZ JHHMMSSS.s+DDMMSS.

To summarize the comparison, we can state that in the common FOV XMM-Newton did not detect (or did not resolve) 87 of the Chandra sources, while Chandra did not detect 24 of the XMM-Newton sources. The first number can be explained mainly by the lower sensitivity and the poor spatial resolution of the XMM-Newton data. The non-detection of the 24 new XMM-Newton sources in the common FOV by Chandra is most likely related to the intrinsic variability of the sources. Investigations of the X-ray variability of young late-type stars on timescales from months to years have found typical variations by factors of 2-4, while some objects show variations of up to factors of $\sim 10$ (e.g. Stauffer et al. 1994; Marino et al. 2003). As nearly all of the new XMM-Newton sources in the Chandra FOV are rather weak sources (PN countrate $<0.01 \mathrm{cnts} / \mathrm{s})$, variations by factors of $\sim 4$ can explain the non-detections in the Chandra data in most cases. The $X M M-N e w t o n$ source list may also contain a few spurious 
Table 1. X-ray sources in IC 348 that were not already detected by Chandra. The source names in the first column consist of the J2000 coordinates. The EPIC-PN count rates are listed in the second column, followed by information about optical and near-infrared counterparts and known membership to the cluster. "noCP" means that no optical/near-infrared counterpart for the source was found. The extinction and bolometric luminosity estimates in the next two columns were generally taken from Luhman et al. (2003). We then list the extinction-corrected $\mathrm{X}$-ray luminosities in the [0.2-10] keV energy band and the fractional X-ray luminosities.

\begin{tabular}{|c|c|c|c|c|c|c|c|c|}
\hline XMMU J & $\begin{array}{c}\text { PN count rate } \\
{[\mathrm{cnts} / \mathrm{s}]}\end{array}$ & Ident. & member & SpT & $\begin{array}{r}A_{J} \\
{[\mathrm{mag}]}\end{array}$ & $\begin{array}{c}L_{\text {bol }} \\
{\left[L_{\odot}\right]}\end{array}$ & $\begin{array}{c}L_{\mathrm{X}} \\
{[\mathrm{erg} / \mathrm{s}]}\end{array}$ & $\log \left(\frac{L_{\mathrm{X}}}{L_{\mathrm{bol}}}\right)$ \\
\hline $034344.5+321616$ & $0.0015 \pm 0.0005$ & noCP & $\mathrm{n}$ & & & & & \\
\hline $034344.5+320816$ & $0.0115 \pm 0.0010$ & L67 & $\mathrm{y}$ & M0.75 & 0.68 & 0.48 & $6.64 \mathrm{e} 29$ & -3.44 \\
\hline $034348.4+321345$ & $0.0029 \pm 0.0006$ & L261 & $\mathrm{y}$ & M5 & 0.28 & 0.06 & $1.07 \mathrm{e} 29$ & -3.33 \\
\hline $034348.7+320733$ & $0.0044 \pm 0.0006$ & L111 & $\mathrm{y}$ & M1.5 & 0.74 & 0.34 & $2.75 \mathrm{e} 29$ & -3.68 \\
\hline $034348.7+321551$ & $0.0064 \pm 0.0008$ & 2MASS & $?$ & & & & & \\
\hline $034348.8+321647$ & $0.0033 \pm 0.0007$ & noCP & $\mathrm{n}$ & & & & & \\
\hline $034349.1+321212$ & $0.0017 \pm 0.0005$ & 2MASS & $?$ & & & & & \\
\hline $034351.7+320453$ & $0.0050 \pm 0.0006$ & noCP & $\mathrm{n}$ & & & & & \\
\hline $034352.1+320343$ & $0.0018 \pm 0.0005$ & 2MASS & $?$ & & & & & \\
\hline $034354.4+321444$ & $0.0020 \pm 0.0005$ & noCP & $\mathrm{n}$ & & & & & \\
\hline $034354.6+320030$ & $0.0029 \pm 0.0006$ & L124 & $\mathrm{y}$ & M4.25 & 0.86 & 0.27 & $2.09 \mathrm{e} 29$ & -3.70 \\
\hline $034355.8+320212$ & $0.0017 \pm 0.0005$ & L26 & $\mathrm{y}$ & K7 & 2.07 & 1.5 & $3.80 \mathrm{e} 29$ & -4.18 \\
\hline $034357.2+321521$ & $0.0027 \pm 0.0006$ & noCP & $\mathrm{n}$ & & & & & \\
\hline $034357.5+320137$ & $0.0030 \pm 0.0005$ & L49 & $\mathrm{y}$ & M0.5 & 2.88 & 0.36 & $1.06 \mathrm{e} 30$ & -3.12 \\
\hline $034358.2+321712$ & $0.0021 \pm 0.0005$ & LHa87 & $\mathrm{y}$ & & & & & \\
\hline $034358.4+320212$ & $0.0020 \pm 0.0005$ & noCP & $\mathrm{n}$ & & & & & \\
\hline $034359.5+321551$ & $0.0032 \pm 0.0006$ & 2MASS & $?$ & & & & & \\
\hline $034359.5+320155$ & $0.0116 \pm 0.0015$ & L13 & $\mathrm{y}$ & M0.5 & 4.13 & 3.2 & $6.67 \mathrm{e} 30$ & -3.27 \\
\hline $034401.1+321736$ & $0.0015 \pm 0.0006$ & noCP & $\mathrm{n}$ & & & & & \\
\hline $034402.1+321940$ & $0.0048 \pm 0.0008$ & 2MASS & $?$ & & & & & \\
\hline $034408.7+321610$ & $0.0145 \pm 0.0010$ & L44 & $\mathrm{y}$ & K0 & 1.00 & 1.7 & $1.24 \mathrm{e} 30$ & -3.72 \\
\hline $034409.2+320355$ & $0.0013 \pm 0.0003$ & L173 & $\mathrm{y}$ & M5.75 & 0.57 & 0.12 & $6.42 \mathrm{e} 28$ & -3.86 \\
\hline $034411.3+321938$ & $0.0098 \pm 0.0010$ & L137 & $\mathrm{y}$ & M3 & 0.52 & 0.20 & $4.51 \mathrm{e} 29$ & -3.23 \\
\hline $034413.8+315534$ & $0.0388 \pm 0.0040$ & 2MASS & $?$ & & & & & \\
\hline $034418.8+322044$ & $0.0113 \pm 0.0011$ & noCP & $\mathrm{n}$ & & & & & \\
\hline $034419.0+321717$ & $0.0192 \pm 0.0012$ & o Per & $?$ & & & & & \\
\hline $034419.7+320903$ & $0.0008 \pm 0.0003$ & 2MASS & $?$ & & & & & \\
\hline $034420.2+321426$ & $0.0015 \pm 0.0004$ & 2MASS & $?$ & & & & & \\
\hline $034422.6+320653$ & $0.0031 \pm 0.0004$ & 2MASS & $?$ & & & & & \\
\hline $034423.4+320427$ & $0.0014 \pm 0.0003$ & noCP & $\mathrm{n}$ & & & & & \\
\hline $034424.6+320643$ & $0.0036 \pm 0.0004$ & L92 & $\mathrm{y}$ & $\mathrm{M} 2.5$ & 0.81 & 0.39 & $2.49 \mathrm{e} 29$ & -3.78 \\
\hline $034425.4+320750$ & $0.0007 \pm 0.0003$ & L630 & $\mathrm{n}$ & & & & & \\
\hline $034426.5+320554$ & $0.0004 \pm 0.0002$ & noCP & $\mathrm{n}$ & & & & & \\
\hline $034426.9+321418$ & $0.0010 \pm 0.0003$ & 2MASS & $?$ & & & & & \\
\hline $034427.9+320519$ & $0.0012 \pm 0.0003$ & L373 & $\mathrm{y}$ & M5.5 & 0.35 & 0.02 & $4.28 \mathrm{e} 28$ & -3.26 \\
\hline $034429.1+320421$ & $0.0008 \pm 0.0003$ & L385 & $\mathrm{y}$ & M5.75 & 0.78 & 0.03 & $5.19 \mathrm{e} 28$ & -3.35 \\
\hline $034429.7+320358$ & $0.0012 \pm 0.0003$ & L295 & $\mathrm{y}$ & M5 & 1.17 & 0.07 & $1.26 \mathrm{e} 29$ & -3.33 \\
\hline $034429.9+321921$ & $0.0030 \pm 0.0006$ & 2MASS & $?$ & & & & & \\
\hline $034430.8+320735$ & $0.0014 \pm 0.0003$ & L207 & $\mathrm{y}$ & M3 & 1.78 & 0.17 & $2.44 \mathrm{e} 29$ & -3.43 \\
\hline $034431.8+320530$ & $0.0006 \pm 0.0003$ & noCP & $\mathrm{n}$ & & & & & \\
\hline $034432.1+320746$ & $0.0013 \pm 0.0003$ & noCP & $\mathrm{n}$ & & & & & \\
\hline $034433.4+321615$ & $0.0009 \pm 0.0004$ & 2MASS & $?$ & & & & & \\
\hline $034434.1+321957$ & $0.0039 \pm 0.0007$ & 2MASS & $?$ & & & & & \\
\hline $034434.6+315758$ & $0.0025 \pm 0.0005$ & noCP & $\mathrm{n}$ & & & & & \\
\hline $034438.7+321905$ & $0.0061 \pm 0.0008$ & 2MASS & $?$ & & & & & \\
\hline $034438.8+315713$ & $0.0011 \pm 0.0004$ & noCP & $\mathrm{n}$ & & & & & \\
\hline $034439.5+321049$ & $0.0006 \pm 0.0003$ & noCP & $\mathrm{n}$ & & & & & \\
\hline $034441.7+321405$ & $0.0006 \pm 0.0003$ & noCP & $\mathrm{n}$ & & & & & \\
\hline $034444.5+321235$ & $0.0009 \pm 0.0003$ & noCP & $\mathrm{n}$ & & & & & \\
\hline
\end{tabular}

Comments: $034425.4+320750=$ L630 is probably a background object (Luhman et al. 2003). 
Table 1. continued.

\begin{tabular}{|c|c|c|c|c|c|c|c|c|}
\hline XMMU J & $\begin{array}{c}\text { PN count rate } \\
{[\mathrm{cnts} / \mathrm{s}]}\end{array}$ & Ident. & member & SpT & $\begin{array}{r}A_{J} \\
{[\mathrm{mag}]}\end{array}$ & $\begin{array}{l}L_{\text {bol }} \\
{\left[L_{\odot}\right]}\end{array}$ & $\begin{array}{c}L_{\mathrm{X}} \\
{[\mathrm{erg} / \mathrm{s}]}\end{array}$ & $\log \left(\frac{L_{\mathrm{X}}}{L_{\mathrm{bol}}}\right)$ \\
\hline $034444.8+322020$ & $0.0026 \pm 0.0006$ & noCP & $\mathrm{n}$ & & & & & \\
\hline $034446.0+321934$ & $0.0013 \pm 0.0005$ & noCP & $\mathrm{n}$ & & & & & \\
\hline $034449.4+321303$ & $0.0007 \pm 0.0003$ & noCP & $\mathrm{n}$ & & & & & \\
\hline $034452.3+315824$ & $0.0036 \pm 0.0006$ & 2MASS & $?$ & & & & & \\
\hline $034452.6+320056$ & $0.0024 \pm 0.0004$ & L1939 & $\mathrm{y}$ & M4.75 & 0.38 & 0.16 & $8.85 \mathrm{e} 28$ & -3.84 \\
\hline $034452.9+320005$ & $0.0015 \pm 0.0004$ & 2MASS & $?$ & & & & & \\
\hline $034454.6+315749$ & $0.0016 \pm 0.0005$ & noCP & $\mathrm{n}$ & & & & & \\
\hline $034455.0+321548$ & $0.0008 \pm 0.0004$ & noCP & $\mathrm{n}$ & & & & & \\
\hline $034501.1+321740$ & $0.0023 \pm 0.0005$ & noCP & $\mathrm{n}$ & & & & & \\
\hline $034501.3+321225$ & $0.0021 \pm 0.0004$ & L201 & $\mathrm{y}$ & M4 & 0.55 & 0.11 & $9.94 \mathrm{e} 28$ & -3.63 \\
\hline $034505.1+315752$ & $0.0027 \pm 0.0006$ & 2MASS & $?$ & & & & & \\
\hline $034507.5+315959$ & $0.0073 \pm 0.0008$ & noCP & $\mathrm{n}$ & & & & & \\
\hline $034507.6+320027$ & $0.0785 \pm 0.0023$ & L1927 & $?$ & $\mathrm{~K} 3$ & & & & \\
\hline $034508.8+315839$ & $0.0059 \pm 0.0007$ & noCP & $\mathrm{n}$ & & & & & \\
\hline $034510.5+321207$ & $0.0019 \pm 0.0004$ & noCP & $\mathrm{n}$ & & & & & \\
\hline $034513.2+320057$ & $0.0018 \pm 0.0005$ & noCP & $\mathrm{n}$ & & & & & \\
\hline $034520.3+320023$ & $0.0019 \pm 0.0005$ & noCP & $\mathrm{n}$ & & & & & \\
\hline $034522.0+320203$ & $0.0029 \pm 0.0006$ & 2MASS & $?$ & & & & & \\
\hline $034530.6+320155$ & $0.0155 \pm 0.0013$ & L10363 & $\mathrm{y}$ & K6 & 0.66 & 0.71 & $8.70 \mathrm{e} 29$ & -3.33 \\
\hline $034532.1+320312$ & $0.0068 \pm 0.0009$ & L10289 & $\mathrm{y}$ & M3 & 0.34 & 0.13 & $2.36 \mathrm{e} 29$ & -3.33 \\
\hline $034535.3+320328$ & $0.0048 \pm 0.0008$ & 2MASS & $?$ & & & & & \\
\hline $034535.9+320733$ & $0.0031 \pm 0.0006$ & noCP & $\mathrm{n}$ & & & & & \\
\hline
\end{tabular}

Comments: 034507.6+320027 = L1927 is an uncertain cluster member (Luhman et al. 2003).

detections among the weakest sources, in particular among the 13 sources without optical/infrared counterpart. Furthermore, we note that the majority of the new XMM-Newton sources is located more than $5^{\prime}$ away from the cluster center, where the sensitivity of the Chandra image is smaller than in the center.

In order to identify the X-ray sources, we compared their coordinates derived from the XMM-Newton data to optical (DSS) and near-infrared images (2MASS) and searched for counterparts in the catalog of IC 348 cluster members by Luhman et al. (2003). We found that 29 of the 71 XMM-Newton sources have neither an optical nor a 2MASS counterpart; we suspect that they are (probably extragalactic) background objects, most likely active galactic nuclei. Among the $42 X M M-N e w t o n$ sources with optical/infrared counterparts, 23 can be identified with known stars (mostly from the lists in Luhman et al. 2003), and 20 of them are known cluster members.

\subsection{Determination of countrates}

With SAS we extracted the counts for the XMM-Newton sources in circular apertures and in the [0.2-10] keV energy band. We generally used aperture radii of $15^{\prime \prime}$, which include $70 \%$ of the point-source flux. For some sources slightly smaller apertures had to be used due to the presence of another nearby source. In all cases we used the appropriate PSF correction. We determined the exposure time for each source from the corresponding exposure maps. The mean source count rates were then computed by subtracting the expected background counts, dividing by the exposure time, and applying the PSF correction factor. The resulting count rates are listed in Table 1. We note that the PN countrates of all sources are far below the level ( $~ 8 \mathrm{cnts} / \mathrm{s})$ above which the "photon pile up" effect can cause problems.

\subsection{X-ray luminosities}

A detailed spectral analysis, from which the plasma temperature and X-ray luminosity can be reliably derived, is only feasible for sources which yield at least a few hundred counts. For the weaker sources, X-ray luminosities can be estimated from the count rates if reasonable estimates of the hydrogen column density and the plasma temperature are available. As the extinction of the young objects in IC 348 varies considerably, we 


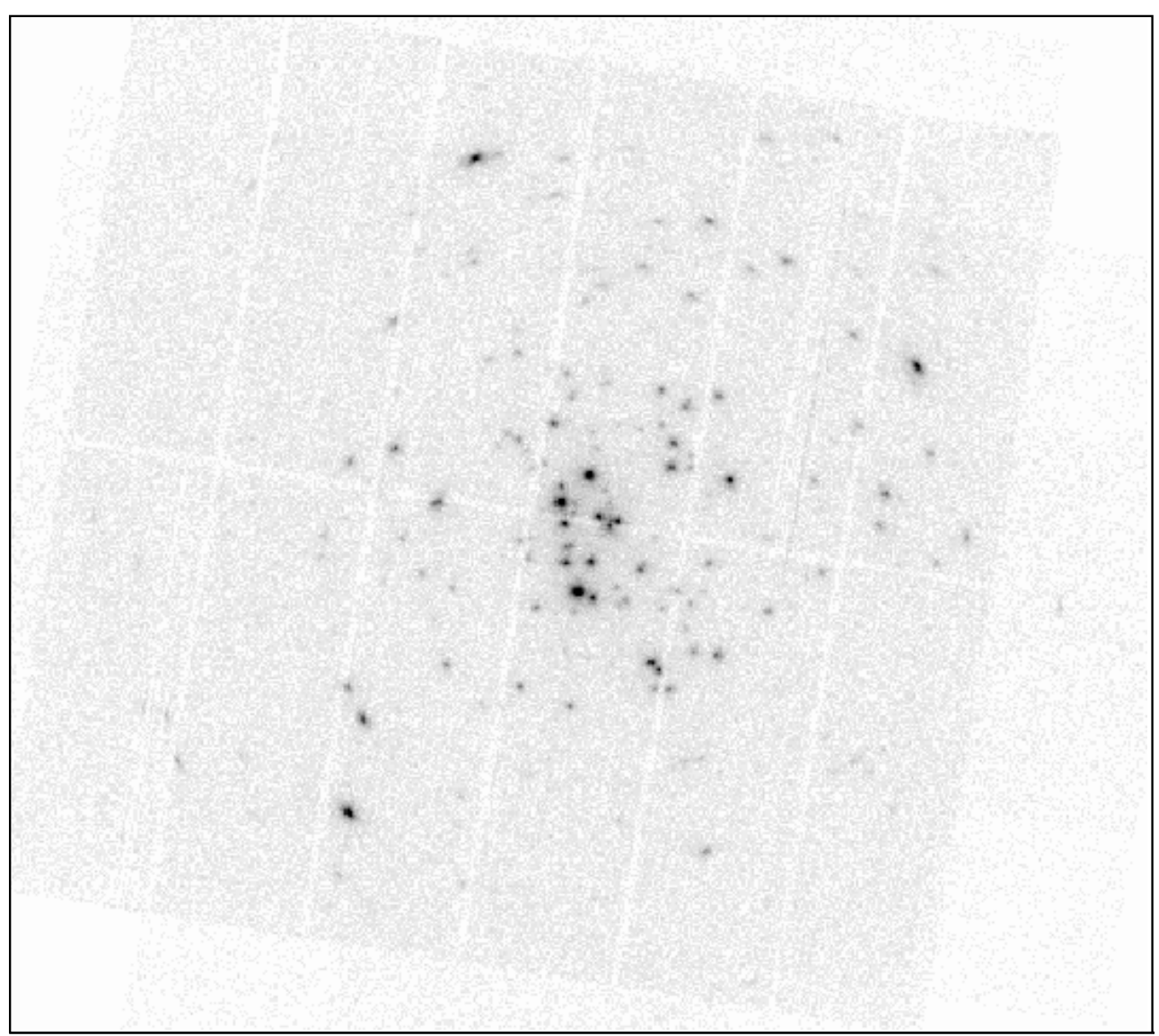

Fig. 2. XMM-Newton image of IC 348. This image is the sum of the MOS1 + MOS2 + PN images, displayed with a square-root intensity scale. The field of view is $\sim 31^{\prime} \times 28^{\prime}$.

calculated X-ray luminosities only for those objects for which extinction estimates are available (mainly from Luhman et al. 2003).

We used the PIMMS ${ }^{1}$ Tool to determine the count rate to X-ray flux ratios for the given extinctions and temperatures. We assumed a plasma temperature of $k T=1 \mathrm{keV}$ (11.6 MK), which is a typical value for young X-ray sources (e.g. Feigelson \& Montmerle 1999; Preibisch 1997; PZ02). The resulting X-ray luminosities (in the [0.2-10] keV band) for the XMM-Newton sources that were not already detected by Chandra range from $4 \times 10^{28} \mathrm{erg} / \mathrm{s}$ to $7 \times 10^{30} \mathrm{erg} / \mathrm{s}$. The corresponding fractional X-ray luminosities $L_{\mathrm{X}} / L_{\mathrm{bol}}$ are between $10^{-4}$ and $10^{-3}$, in the typical range for $\mathrm{T}$ Tauri stars.

In our Chandra data we could detect objects with luminosities as small as $\sim 1 \times 10^{28} \mathrm{erg} / \mathrm{s}$, i.e. our Chandra data are considerably more sensitive than the XMM-Newton data. The reasons for this difference in sensitivity is, first, the shorter useful exposure time of our XMM-Newton data (due to the background flare), and, second, the larger PSF, due to which considerably more photons are required for a significant detection of a point source.

${ }^{1}$ PIMMS is the Portable, Interactive Multi-Mission Simulator provided by the HEASARC Online Service; for further information see http://heasarc.gsfc.nasa.gov/Tools/w3pimms.html

\section{Temporal variability}

With SAS we extracted the lightcurves for all XMM-Newton sources and from a large, source-free, background region. Visual inspection of the individual background-subtracted lightcurves showed irregular, small-amplitude count rate variations in many sources. Only for eight sources did we find significant count rate variations; their lightcurves are shown in Fig. 3. Some of these objects show typical flares with rise and decay times of a few hours, very similar to flares on other X-ray active stars (e.g. Montmerle et al. 1983; Gagné et al. 1995; Preibisch \& Neuhäuser 1995). The ROSAT observations of IC 348 (Preibisch et al. 1996) already showed several large $\mathrm{X}$-ray flares in IC 348, among them a giant X-ray flare on the classical T Tauri star LH $\alpha 92$ (Preibisch et al. 1993), and our Chandra observation of IC 348 (PZ02) revealed strong variability and flares on at least 18 sources.

We note that all the flares seen with XMM-Newton have amplitudes (ratio of maximum count rate to pre-flare count rate) of less than $\sim 3$. This is probably the consequence of the rather short (useful) exposure time of our XMM-Newton observation and the fact that low-amplitude flares are much more frequent than large flares.

In two sources, CXOPZ 034422.3+321201 and $034436.9+320645$, we see a rather slow increase of the 

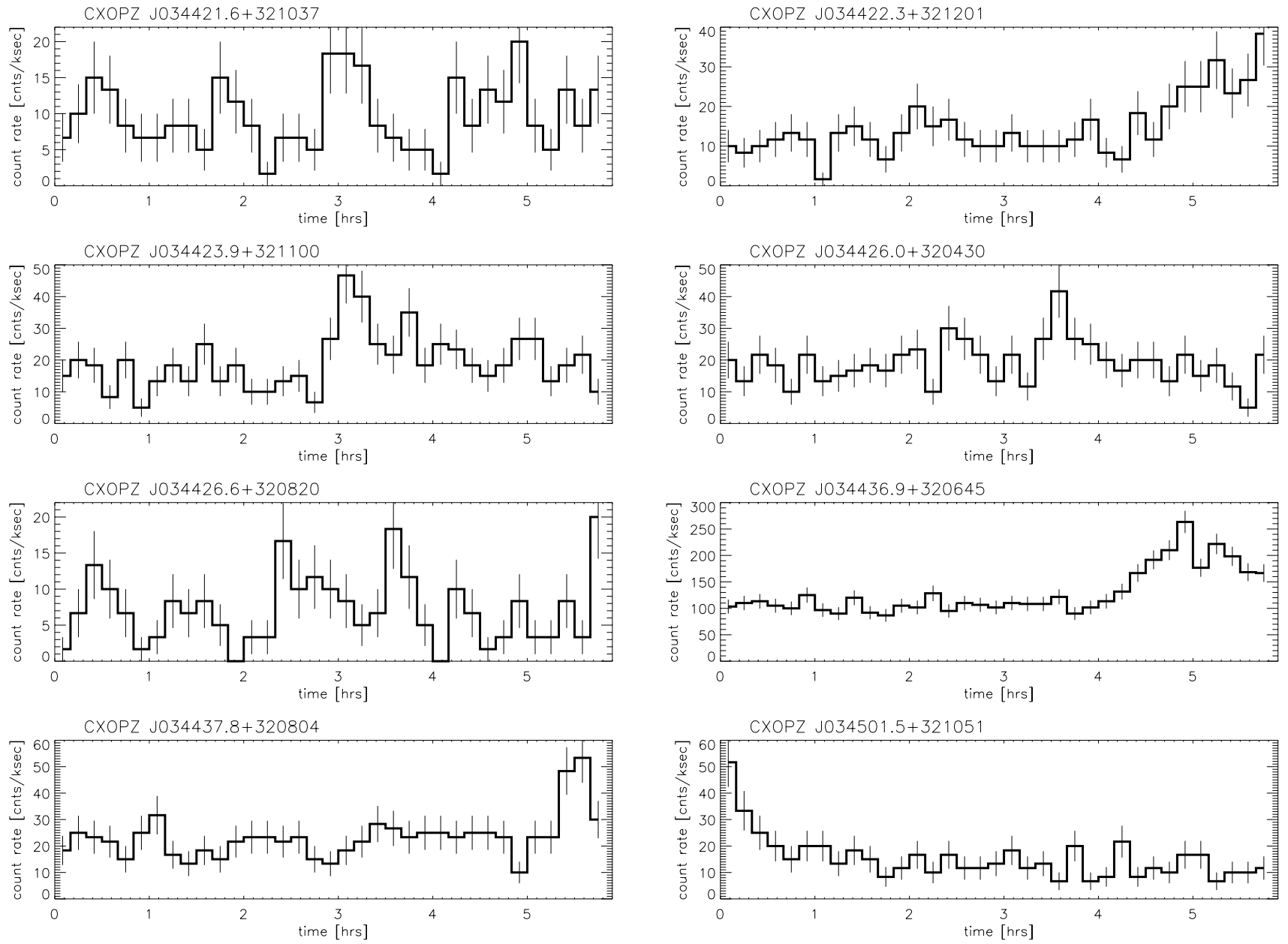

Fig. 3. EPIC-PN lightcurves (background-subtracted) for sources exhibiting significant variability during our observation.

count rate which is probably caused by rotational modulation rather than by a flare.

\section{X-ray spectra}

\subsection{Spectral extraction and fitting}

For those 12 sources with at least 500 source counts we performed a spectral analysis. Eleven of these had already been detected by Chandra. With SAS we extracted the pulse height spectra for each of these sources and for suitable background regions; then we built the corresponding redistribution matrix files and ancillary response files appropriate for the position and size of the source extraction region. We grouped the spectra with 25 counts per bin and performed spectral fits with the Sherpa package contained in CIAO. We used the XSPEC model "raymond" describing the emission from a thermal plasma spectrum and "wabs" for the absorption model.

We first tried to fit the spectra with a single-temperature plasma model plus absorption, but this model did not yield statistically acceptable fits in most cases. We therefore used a two-temperature model (2T model hereafter), which gave statistically acceptable fits to all the spectra. The fitting results (plasma temperatures and hydrogen column density) are shown in Figs. 5 and 6. The plasma temperatures found in the fits are in the typical range for young stellar X-ray sources (e.g. Feigelson \& Montmerle 1999; Preibisch 1997; PZ02).

We note that two of our strong X-ray sources, CXOPZ J034435.3+321005 and J034450.7+321904, are A-type stars, from which one would not expect coronal X-ray emission as observed from late-type (G-, K-, and M-type) $\mathrm{T}$ Tauri stars. As discussed in detail in PZ01, the X-ray emission from these objects originates most likely not from the A-type stars, but from unresolved late-type T Tauri star companions (see also Stelzer et al. 2003).

\subsection{Comparison of XMM-Newton and Chandra spectral fitting results}

\subsubsection{Plasma temperatures}

It is clear that the $2 \mathrm{~T}$ model we used for our spectral fitting analysis (and which is frequently used in similar studies) cannot be strictly correct, because the coronae of active stars are generally known to show a broad, continuous distribution of plasma temperatures (e.g. Brickhouse et al. 2000; Sanz-Forcada et al. 2003). Given the relatively low numbers of counts per spectrum, a more detailed spectral analysis is hardly warranted for our data. The meaning of the two temperature values derived in the spectral fits is therefore not fully clear. Do the derived 

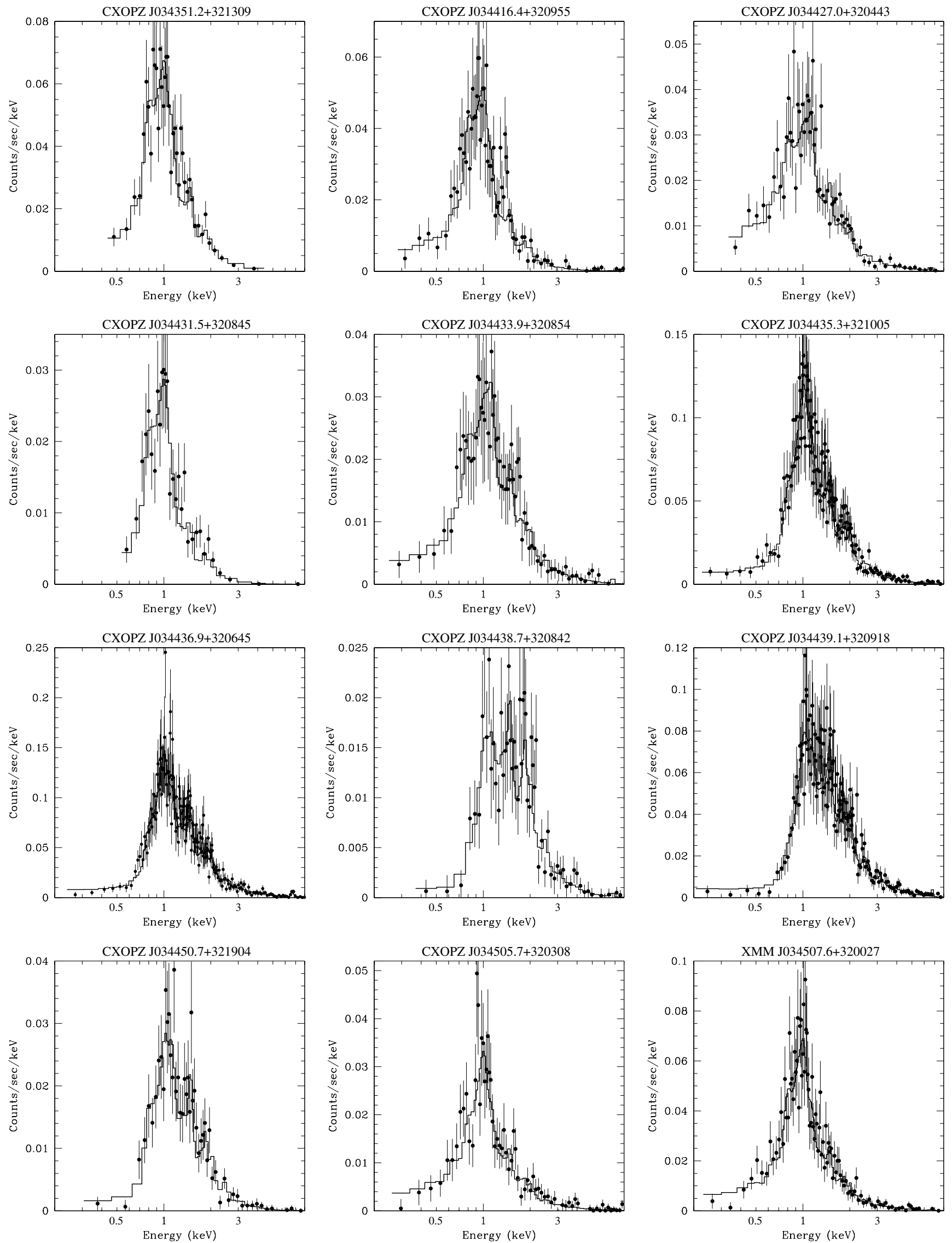

Fig. 4. XMM-Newton-EPIC-PN spectra of the 12 sources with more than 500 detected counts. The solid dots with error bars show the observed spectrum, the histogram shows the best fit model. 


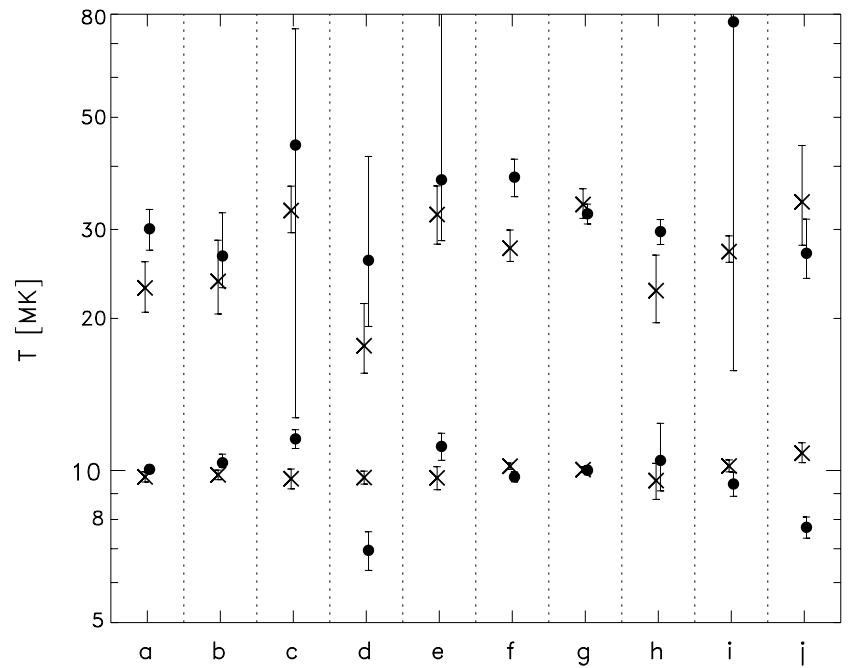

Fig. 5. Comparison of the plasma temperatures derived from the fits to the XMM-Newton and Chandra spectra for 10 stars in IC 348. The two temperature components found from the XMM-Newton spectra for each star (labeled as $a, b, c, \ldots$ ) are plotted with $X$ signs, the two temperature components found from the Chandra spectra for each star by the solid dots. The following objects are shown: a: CXOPZ J034351.2+321309; b: CXOPZ J034416.4+320955; c: CXOPZ J034427.0+320443; d: CXOPZ J034431.5+320845; e: CXOPZ J034433.9+320854; f: CXOPZ J034435.3+321005; g: CXOPZ J034436.9+320645; h: CXOPZ J034438.7+320842; i: CXOPZ J034439.1+320918; j: CXOPZ J034505.7+320308.

temperatures correspond to peaks of the underlying temperature distribution (see e.g. Peres et al. 2000), or do they reflect just those values of the continuous temperature distribution for which the detector is most sensitive?

We can get some insight into this problem by comparing the fitting results for spectra of the same sources observed with different instruments. There are $10 \mathrm{X}$-ray sources in IC 348 for which we have good spectra from both XMM-Newton and Chandra. The comparison of the $2 \mathrm{~T}$ model fitting results for these sources can give us information on the spectral variability of the stars and the reliability of the extracted spectral information. In Fig. 5 we compare the plasma temperatures derived from the 2T model fits to the XMM-Newton and the Chandra spectra of these sources.

The first thing to note is that the temperatures derived from the Chandra and XMM-Newton spectra agree generally quite well. With only very few exceptions, the temperatures agree within the " $1 \sigma$ " error intervals. This suggests that the derived temperatures correspond to some real structures in the coronal plasma temperature distributions of these stars.

Another interesting result of our comparison is the remarkable similarity of the low-temperature components in our sample. For all stars a temperature of about $10 \mathrm{MK}$ is found from the XMM-Newton as well as the Chandra spectra. This suggests that this cool component in the $2 \mathrm{~T}$ model fits corresponds to a real feature in the coronal temperature distribution of the $\mathrm{T}$ Tauri stars, at a characteristic temperature of $10 \mathrm{MK}$. It is interesting to note that a $\sim 10 \mathrm{MK}$ plasma component seems to be some kind of a general feature of coronally active stars; for

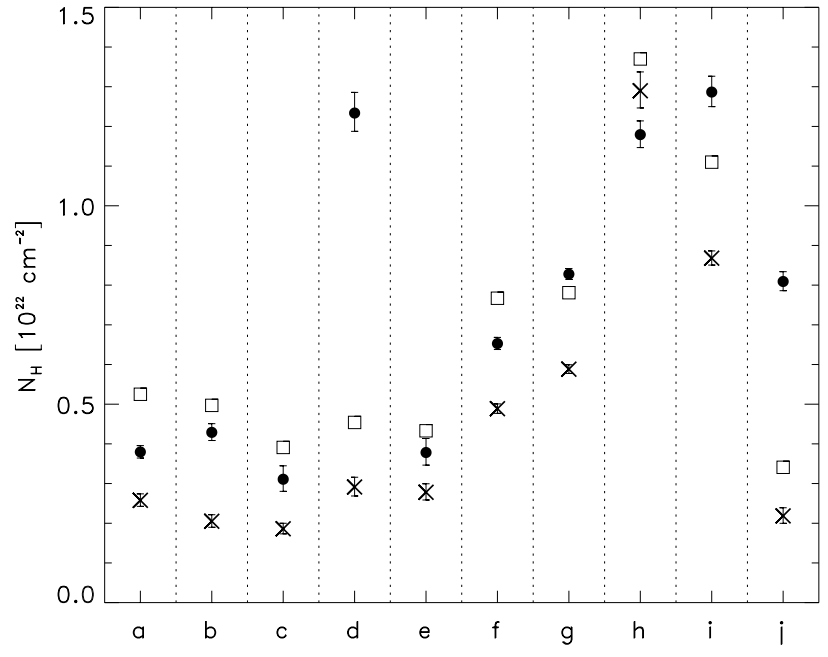

Fig. 6. Comparison of the hydrogen column densities derived from the fits to the XMM-Newton spectra (X) and the Chandra spectra (solid dots) for 10 stars in IC 348. The objects are the same as those shown in Fig. 5. The open boxes show the $N_{\mathrm{H}, \mathrm{IR}}$ values computed from the nearinfrared extinction of the stars as determined by Luhman et al. (2003). Note that the Chandra spectrum of source (d) had rather poor S/N and contained no photons with energies below $0.8 \mathrm{keV}$; this has apparently caused a large overestimate of the extinction.

example, Sanz-Forcada et al. (2003) determined the emission measure distribution of 28 coronally active stars, and found that in most stars the emission measure distribution shows a peak at 8-10 MK. They argued that this temperature component defines a fundamental coronal structure, which is probably related to a class of compact loops with high plasma density.

The fitted values for the hot plasma components, on the other hand, show a much wider spread from star to star, from $\sim 20 \mathrm{MK}$ to $\sim 50 \mathrm{MK}$. Remarkably, in eight of the ten stars the temperatures derived from the Chandra and the XMM-Newton spectra agree within the " $1 \sigma$ " error. This suggests that the thermal structure of the coronal plasma of these stars did not change significantly over the $\sim 2.4$ year time period between our Chandra and the XMM-Newton observation. The temperature of the dominant coronal structure must therefore be rather constant over several years. It has been argued that the hot temperature component in the spectra of active stars may be related to continuous flaring activity (e.g. Kashyap et al. 2002). The lightcurves of the sources we investigated here show no strong flares, but an apparently even lightcurve may in fact be a superposition of numerous flares. If the rise and decay phases of the individual events overlap strongly enough, only small count rate fluctuations would be observed, which would be hard to distinguish from random Poisson counting fluctuations. Applying this scenario to the stars studied here, one would conclude that the distribution of flare properties, especially the temporal averages of the flare plasma temperatures, would have to be rather constant over periods of several years.

\subsubsection{Hydrogen column densities}

Finally, it is also interesting to look at the hydrogen column densities derived in the spectral fits. Figure 6 shows the 
fitted values of $N_{\mathrm{H}}$ and also the values $N_{\mathrm{H}, \mathrm{IR}}$ computed from the near-infrared extinction $A_{J}$ given by Luhman et al. (2003) using the relations $N_{\mathrm{H}}=A_{\mathrm{V}} \times 2 \times 10^{21} \mathrm{~cm}^{-2}$ and $A_{\mathrm{V}}=3.55 \times$ $A_{J}$. Here we see that the fits to the Chandra spectra yield systematically higher values for $N_{\mathrm{H}}$ than those derived from the XMM-Newton spectra. This difference may be related to the enhanced low-energy absorption due to contamination on the ACIS chips, which could lead to overestimation of the $N_{\mathrm{H}}$ values derived from Chandra spectra (see discussion in Favata et al. 2003). If, however, we consider the $N_{\mathrm{H}, \mathrm{IR}}$ values derived from the near-infrared extinction, we find that in all cases the fits to the XMM-Newton spectra gave too low (typically by a factor of 2) values of $N_{\mathrm{H}}$, while the values from the fits to the Chandra spectra are in most cases closer to $N_{\mathrm{H}, \mathrm{IR}}$.

It remains unclear whether the Chandra or the $X M M$-Newton spectra provide a better estimate of the $\mathrm{X}$-ray extinction. A more detailed analysis of this effect would require spectra with higher $\mathrm{S} / \mathrm{N}$ and is beyond the scope of this paper.

\section{The X-ray emitting young stellar population in IC 348}

\subsection{The new $X$-ray sources and the $T$ Tauri star population of IC 348}

As already noted above, 42 of the new XMM-Newton sources have optical or infrared counterparts and can therefore be regarded as potential cluster members. 20 sources can be directly identified with known cluster members; most of them are of spectral type $\mathrm{M}$.

Three sources are identified with objects for which membership of the cluster is unclear. The first of these is XMMU J034425.4+320750; it corresponds to star \# 630 in Luhman et al. (2003), who classified it as a likely early type or giant background star; since the X-ray source is very weak we cannot investigate its X-ray properties in detail to check whether they are consistent with that classification. The second one is the rather strong source XMMU J034507.6+320027, which is identified with star \# 1927 in Luhman et al. (2003), who classified it as an uncertain member of spectral type K3. The fit to the X-ray spectrum (see Fig. 4) yields $N_{\mathrm{H}}=2.4 \times$ $10^{21} \mathrm{~cm}^{-2}, T_{1}=10 \mathrm{MK}, T_{2}=34 \mathrm{MK}$; assuming that the object is at the distance of IC 348 , we derive an X-ray luminosity of $L_{X}=7.5 \times 10^{30} \mathrm{erg} / \mathrm{s}$. These X-ray properties are very similar to those of similar $\mathrm{T}$ Tauri cluster members and therefore favor the possible membership of this star of IC 348. The third object is the optically bright star $o$ Per, which will be discussed in detail below.

Furthermore, 19 of the new XMM-Newton sources have relatively bright optical and/or near-infrared counterparts; these objects are new likely cluster members, but further investigations are necessary to establish their membership status. Most of these objects are located in the outskirts of the cluster, more than $5^{\prime}$ away from the center. Their X-ray emission suggests that they are young low-mass stars with masses between about $1.5 M_{\odot}$ and $\sim 0.1 M_{\odot}$.

\subsection{The B1 III star o Per}

$o$ Per $(=$ HD 23180) is by far the brightest $(V=3.8)$ star in the area of the IC 348 cluster. The Hipparcos parallax is $\pi=$ $(2.21 \pm 0.84)$ mas, translating to a distance estimate of $452 \mathrm{pc}$ within a range of 330-730 pc, leaving its relation to the cluster unclear. Fredrick (1956) rejected $o$ Per from membership of the cluster because of its large proper motion. Two recent studies of the space motions of the stars in the Perseus OB2 association, based on Hipparcos data (de Zeeuw 1999) and on radial velocities (Steenbrugge et al. 2003), also suggested that $o$ Per is probably not a member of Perseus OB2 and therefore also probably not related to IC 348. $o$ Per is a spectroscopic binary with a period of 4.4 days (Lynds 1960). The primary is a B1 III star, the secondary is probably a B1 V star (Foy 1972). Morris (1985) analyzed the lightcurve and spectroscopic information for the system and derived stellar masses of $\sim 9 M_{\odot}$ for the primary and $\sim 6 M_{\odot}$ for the secondary. The Hipparcos observations of $o$ Per revealed the presence of a visual companion at a separation of $1.02^{\prime \prime}$ and with a magnitude difference of $2.91 \mathrm{mag}$. Speckle interferometry by Mason et al. (2001) confirmed this detection. If this visual companion is physically related to $o$ Per, and if we assume it to be a main-sequence star, its magnitude suggests a spectral type of $\sim \mathrm{B} 4$ and an estimated mass of $\sim 4-6 M_{\odot}$. It therefore seems that $o$ Per consists of three intermediate mass stars. Assuming a distance of $D=450 \mathrm{pc}$ as suggested by the Hipparcos measurements, we estimate a luminosity of the primary component in $o$ Per of $L_{\mathrm{bol}}=16000 L_{\odot}$.

$o$ Per is clearly detected as an X-ray source in our $X M M$-Newton image. This is interesting because intermediatemass stars are expected to lack the magnetically driven coronae common among low-mass stars. Although our X-ray spectrum for $o$ Per contains only 284 source counts, we performed a spectral analysis (see Fig. 7). The fit gives $N_{\mathrm{H}}=$ $2.0 \times 10^{21} \mathrm{~cm}^{-2}$, which agrees very well with the extinction of $A_{\mathrm{V}}=0.96 \mathrm{mag}$ derived by Snow et al. (1994). The derived temperatures are $T_{1}=(2.8 \pm 0.1) \mathrm{MK}$, and $T_{2}=$ $16.2_{-0.2}^{+0.4} \mathrm{MK}$, with most of the emission measure associated with the low-temperature component $\left(E M_{1}: E M_{2}=1.8: 1\right)$. This temperature structure is clearly different from that of the $\mathrm{T}$ Tauri stars described in the last section, since the dominant low-temperature component is much cooler than found in the $\mathrm{T}$ Tauri stars. The X-ray luminosity computed from the fit parameters is $L_{\mathrm{X}}=5.3 \times 10^{30} \mathrm{erg} / \mathrm{s}$ and gives $L_{\mathrm{X}} / L_{\mathrm{bol}} \approx 9 \times 10^{-8}$. The X-ray lightcurve of $o$ Per shows no evidence for significant variability.

The fractional X-ray luminosity, the softness of the spectrum, and the absence of X-ray flares are in good agreement with the expectations of the radiation-driven wind shock model for O and very early B stars (see e.g. Lucy \& White 1980; Casinelli et al. 1994; Casinelli et al. 2001; Kahn et al. 2001; Schulz et al. 2003). However, the wind shock model would not predict the observed hot plasma component of $T_{2} \sim 16 \mathrm{MK}$. The derived plasma temperature structure of $o$ Per is to some degree similar to that of the early B-type stars in the Orion Nebula Cluster derived by Schulz et al. (2003): in addition to the rather cool plasma component typical for B-stars and probably related to wind shocks, these stars also show a high plasma 


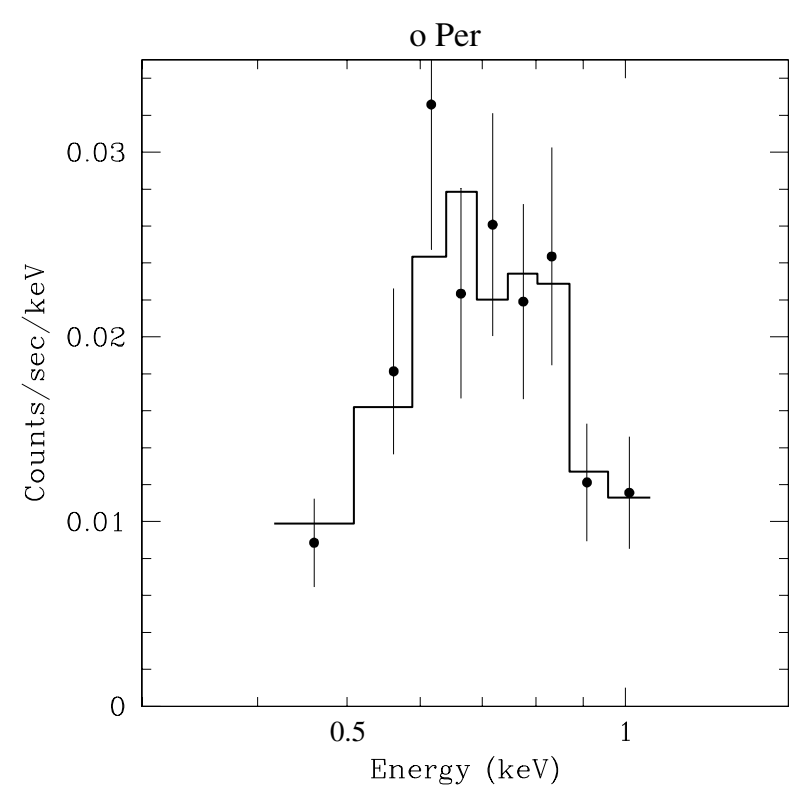

Fig. 7. The XMM-Newton-EPIC-PN spectrum of $o$ Per. The solid dots with error bars show the observed spectrum, the histogram shows the best fit model. Note that the spectrum is quite soft and contains no photons with energies above $1.2 \mathrm{keV}$.

temperature component, which largely exceeds the temperatures predicted by the canonical wind shock models. Schulz et al. (2003) used high-resolution grating X-ray spectra to study the thermal structure in detail and concluded that the hot plasma component is likely the result of magnetic confinement and suggests the presence of an active corona. Since this kind of magnetic activity is only observed in very young ( $\$ 1 \mathrm{Myr}$ ) OB stars, it could possibly be of pre-main sequence origin, i.e. due to primordial magnetic fields. This hypothesis would suggest that $o$ Per is a very young object.

However, with the data at hand we cannot know which of the (at least) three optical components of $o$ Per is the X-ray source. Furthermore, we cannot exclude the presence of additional, yet undetected, low-mass companions. One alternative explanation for the observed X-ray properties of $o$ Per would therefore be that one (presumably the most luminous) of the B-type stars produces the soft component of the X-ray flux by wind shocks, and a hypothetical low-mass companion would produce the harder X-ray component by coronal emission. From our spectral fit we find the X-ray luminosity of the hot plasma component to be $\sim 1 \times 10^{30} \mathrm{erg} / \mathrm{s}$. Such high X-ray luminosities are only reached by the most active main-sequence stars, but found for a significant fraction of $\mathrm{T}$ Tauri stars. It is therefore another hint towards the youth of the $o$ Per.

\subsection{Brown dwarfs}

During the last few years, X-ray emission has been detected from several brown dwarfs (e.g. Neuhäuser \& Comerón 1998; Rutledge et al. 2000; Garmire et al. 2000; Imanishi et al. 2001). Most of the X-ray detected brown dwarfs are very young objects with ages of at most $10^{7}$ years, and all are still relatively warm, with late $\mathrm{M}$ spectral types. Their typical fractional X-ray luminosities are $\left(L_{\mathrm{X}} / L_{\mathrm{bol}}\right) \sim 10^{-4}-10^{-3}$, i.e. very similar to the values observed for active very-low mass stars.

In IC 348, our Chandra observation led to the discovery of weak X-ray emission from 7 of the brown dwarfs and brown dwarf candidates (PZ01; PZ02) with X-ray luminosities between $8 \times 10^{27} \mathrm{erg} / \mathrm{s}$ and $3.6 \times 10^{28} \mathrm{erg} / \mathrm{s}$.

The recent census of the stellar and substellar members of IC 348 by Luhman et al. (2003) has raised the total number of spectroscopically identified brown dwarfs in IC 348 to 23. We searched for X-ray counterparts of these brown dwarfs, but could detect none of them as X-ray sources in our $X M M-N e w t o n$ data. These non-detections can be understood as a consequence of the sensitivity limit of our XMM-Newton data ( $L_{\mathrm{X}, \mathrm{lim}} \sim 4 \times 10^{28} \mathrm{erg} / \mathrm{s}$ for objects with low extinction), which is just above the luminosity of the brightest IC 348 brown dwarfs detected with Chandra. Only due to its $\sim 4 \times$ higher point-source sensitivity were we able to detected these objects in our Chandra observation.

\subsection{Embedded young stellar objects}

Since X-rays with energies above $\sim 1 \mathrm{keV}$ are much less affected by extinction than optical light, $\mathrm{X}$-ray observations allow a deep look into dense molecular cloud cores towards deeply embedded young stellar objects (e.g. Feigelson \& Montmerle 1999). While most members of IC 348 are optically visible $\mathrm{T}$ Tauri stars, the dense cloud core south-west of the cluster center contains several deeply embedded infrared sources, including at least two jet and outflow sources (see Eislöffel et al. 2003). The most prominent objects are the very young molecular hydrogen jet HH 211 (McCaughrean et al. 1994) and the bright infrared source IC 348-IR (Strom et al. 1974), also known as "flying ghost nebula" (Boulard et al. 1995). These objects were outside the field-of-view of our Chandra image.

Two of the embedded objects in this area are detected as X-ray sources in our XMM-Newton image. XMMU J034359.5+320155 can be identified with the "flying ghost nebula". This object show a remarkable bipolar nebulosity which indicates that a young stellar object is embedded in a flattened circumstellar structure. Boulard et al. (1995) studied this object and concluded that the young star is surrounded by a circumstellar disk seen nearly edge-on. They analyzed the broad-band spectral energy distribution and claimed that the central source is a young B7.5 (4 $\left.M_{\odot}\right)$ star obscured by $\sim 30$ mag of visual extinction. If this were correct, the object would be the second-most massive young star in IC 348. However, this mass-estimate is in strong contrast to the near-infrared spectroscopy performed by Luhman et al. (1998; star \# 13), which showed that the star is actually of spectral type M0.5 rather than B. This object is therefore not an intermediate-mass star, but a very young low-mass star with $M_{\star} \sim 0.6 M_{\odot}$ and an extinction of $A_{\mathrm{V}} \sim 15 \mathrm{mag}$. We estimate $L_{\mathrm{X}}=6.7 \times 10^{30} \mathrm{erg} / \mathrm{s}$ and $\log \left(L_{\mathrm{X}} / L_{\mathrm{bol}}\right)=-3.3$, in the typical range for TTS.

The other X-ray detected object in this area, XMMU J034357.5+320137, is the strongly obscured $\left(A_{\mathrm{V}} \sim 10 \mathrm{mag}\right) \mathrm{M} 0.5$ star L-49, for which we derive an X-ray 
luminosity of $L_{\mathrm{X}}=1.1 \times 10^{30} \mathrm{erg} / \mathrm{s}$ and $\log \left(L_{\mathrm{X}} / L_{\mathrm{bol}}\right)=-3.1$. We note that Avila et al. (2001) detected a variable $3.5 \mathrm{~cm}$ radio source, called VLA 3, very close to L-49. They suggested that VLA 3 may be the driving source of the north-south outflow seen in molecular hydrogen images of that region. However, Eislöffel et al. (2003) found that the mm source IC 348 MMS and not VLA 3 seems to drive this flow.

Two further X-ray sources in this region are identified with relatively bright near-infrared sources (L26 and L124). The obscured area contains another X-ray source without optical/infrared counterpart, XMMU J034358.4+320212; this source cannot be identified with any of the known embedded objects, and we therefore believe that it is probably an (extragalactic) background source.

Neither the jet source HH 211-mm nor the outflow source IC 348 MMS, both of which are presumably class 0 protostars (Eislöffel et al. 2003) are detected in our XMM-Newton image. This is presumably due to the very high extinction to these objects, which are invisible even in deep $K$-band images.

In our Chandra image of IC 348 we had discovered $\mathrm{X}$-ray emission from two deeply embedded objects, presumably class I protostars, south of the cluster center, L-51 and 2MASS $034443.3+320131$. The class I candidate L-51 is undetected in our XMM-Newton image. The infrared source 2MASS $034443.3+320131$ is marginally detected as a very weak, tentative source, but the number of source photons is too small for analysis of its X-ray properties.

\section{Summary and conclusions}

We have analyzed a deep XMM-Newton observation of the young stellar cluster IC 348. Our previous study of IC 348 with Chandra had led to the detection of $215 \mathrm{X}$-ray sources. The XMM-Newton data have now revealed 71 new X-ray sources, 24 of which are located in the field-of-view of the Chandra image. 20 of the new X-ray sources can be identified with known cluster members, and further 19 sources are likely new lowmass members, mostly located in the outskirts of the cluster. Our main aim, i.e. a significant extension of X-ray detections among the faintest $\mathrm{T}$ Tauri stars, brown dwarfs, or embedded young stellar objects in IC 348, could, however, not be reached, mostly due to the strong background flare which seriously reduced the expected sensitivity of our XMM-Newton data by about a factor of 2. Further problems are caused by the rather poor PSF, which does not allow the resolving of the individual sources in the crowded cluster center.

The newly detected objects display X-ray properties which are in the typical range of the previously known sources in IC 348 and in other star forming regions. None of the new sources shows any particularly remarkable properties, like for example large X-ray flares.

The comparison of the plasma temperature derived from spectral fits to the Chandra and the XMM-Newton spectra of the strongest sources in IC 348 reveals a remarkable consistency of the derived temperatures and suggests that the thermal structure in the coronae of the T Tauri stars can remain essentially constant over periods of several years.
Acknowledgements. We would like to thank the referee, Kevin Luhman, for his prompt and constructive report, which helped to improve this paper. This work is based on observations with $X M M-N e w t o n$, an ESA Science Mission with instruments and contributions directly funded by ESA member states and the USA (NASA). This publication makes use of data products from the Two Micron All Sky Survey, which is a joint project of the University of Massachusetts and the Infrared Processing and Analysis Center, funded by the National Aeronautics and Space Administration and the National Science Foundation.

\section{References}

Avila, R., Rodriguez, L. F., \& Curiel, S. 2001, Rev. Mex. Astron. Astrofis., 37, 201

Boulard, M.-H., Caux, E., Monin, J.-L., Nadeau, D., \& Rowlands, N. 1995, A\&A, 300, 276

Brickhouse, N. S., Dupree, A. K., Edgar, R. J., et al. 2000, ApJ, 530, 387

Casinelli, J. P., Cohen, D. H., MacFarlane, J. J., Sanders, W. T., \& Welsh, B. J. 1994, ApJ, 421, 705

Casinelli, J. P., Miller, N. A., Waldron, W. L., MacFarlane, J. J., \& Cohen, D. H. 2001, ApJ, 554, L55

Cernis, K. 1990, Ap\&SS 166, 315

Eislöffel, J., Fröbrich, D., Stanke, T., \& McCaughrean, M. J. 2003, Ap, 595, 259

Favata, F., Giardion, G., Micela, G., Sciortino, S., \& Damiani, F. 2003, A\&A, 403, 187

Feigelson, E. D., \& Montmerle, T. 1999, ARA\&A, 37, 363

Foy, R. 1972, A\&A, 16, 108

Fredrick, L. W. 1956, AJ, 61, 437

Gagné, M., Caillault, J.-P., \& Stauffer, J. R. 1995, ApJ, 450, 217

Garmire, G., Feigelson, E. D., Broos, P., et al. 2000, AJ, 120, 1426

Herbig, G. H. 1998, ApJ, 497, 736

Imanishi, K., Tsujimoto, M., \& Koyama, K. 2001, ApJ, 563, 361

Jansen, F., Lumb, D., Altieri, B., et al. 2001, A\&A, 365, L1

Kahn, S. M., Leutenegger, M., Cottam, J., et al. 2001, A\&A, 365, L312

Kashyap, V. L., Drake, J. J., Güdel, M., \& Audard, M. 2002, ApJ, 580, 1118

Lada, E. A., \& Lada, C. J. 1995, AJ, 109, 1682

Lada, E. A., Lada, C. J., \& Muench, A. 1998, in The Stellar Initial Mass Function, ed. G. Gilmore, \& D. Howell, ASP Conf. Ser., 142,107

Lucy, L. B., \& White, L. R. 1980, ApJ, 241, 300

Luhman, K. L. 1999, ApJ, 525, 466

Luhman, K. L., Rieke, G. H., Lada, C. J., \& Lada, E. A. 1998, ApJ, 508,347

Luhman, K. L., Stauffer, J. R., Muench, A. A., et al. 2003, ApJ, 593, 1093

Lynds, C. R. 1960, ApJ, 131, 122

Marino, A., Micela, G., Peres, G., \& Sciortino, S. 2003, A\&A, 406, 629

Mason, B. D., Hartkopf, W. I., Holdenried, E. R., \& Rafferty, T. J. 2001, AJ, 121, 3224

McCaughrean, M. J., Rayner, J. T., \& Zinnecker, H. 1994, ApJ, 436, L189

Montmerle, T., Koch-Miramond, L., Falgarone, E., \& Grindlay, J. E. 1983, ApJ, 269, 182

Morris, S. L. 1985, ApJ, 295, 143

Muench, A. A., Lada, E. A., Lada, C. J., et al. 2003, AJ, 125, 2029

Najita, J. R., Tiede, G. P., \& Carr, J. S. 2000, ApJ, 541, 977

Neuhäuser, R., \& Comeron, F. 1998, Science, 282, 83 
Peres, G., Orlando, S., Reale, F., Rosner, R., \& Hudson, H. 2000, ApJ, Schulz, N. S., Canizares, C., Huenemoerder, D., \& Tibbets, K. 2003, 528,537

Preibisch, Th. 1997, A\&A, 324, 690

Preibisch, Th., \& Neuhäuser, R. 1995, in Flares and Flashes, ed. J. Greiner, H. Duerbeck, \& R. E. Gershberg, IAU Coll., 151, 212

Preibisch, Th., \& Zinnecker, H. 2001, AJ, 122, 866

Preibisch, Th., \& Zinnecker, H. 2002, AJ, 123, 1613

Preibisch, Th., Zinnecker, H., \& Schmitt, J. H. M. M. 1993, A\&A, 279, L33

Preibisch, Th., Zinnecker, H., \& Herbig, G. H. 1996, A\&A, 310, 456

Preibisch, Th., Stanke, Th., \& Zinnecker H. 2003, A\&A, 409, 147 ApJ, 595, 365

Snow, T. P., Hanson, M. M., Seab, C. G., \& Saken, J. M. 1994, ApJ, 420,632

Stauffer, J. R., Caillault, J.-P., Gagne, M., Prosser, C. F., \& Hartmann, L. W. 1994, ApJS, 91, 625

Steenbrugge, K. C., de Brunijne, J. H. J., Hoogerwerf, R., \& de Zeeuw, P. T. 2003, A\&A, 402, 587

Stelzer, B., Huelamo, N., Hubrig, S., Zinnecker, H., \& Micela, G. 2003, A\&A, 407, 1067

Strom, S. E., Strom, K. M., \& Carrasco, L. 1974, PASP, 86, 798

Strüder, L., Briel, U., Dennerl, K., et al. 2001, A\&A, 365, L18

Turner, M. J. L., Abbey, A., Arnaud, M., et al. 2001, A\&A, 365, L27 538, L141

de Zeeuw, P. T., Hoogerwerf, R., de Bruijne, J. H. J., Brown, A. G. A., 145, 147 \& Blaauw, A. 1999, AJ, 117, 354 